\author{
Supporting Information
}

\title{
Unveiling interaction potential surface between drug-entrapped polymeric micelles clarifying the high drug nanocarrier efficiency
}

Takeshi Morita, ${ }^{1 *}$ Sayaka Mukaide, ${ }^{2}$ Ziqiao Chen, ${ }^{2}$ Kenjirou Higashi, ${ }^{2 *}$ Hiroshi Imamura, ${ }^{3}$ Kunikazu Moribe, ${ }^{2}$ and Tomonari Sumi* ${ }^{4 *}$

${ }^{1}$ Graduate School of Science, Chiba University, Chiba 263-8522, Japan

${ }^{2}$ Graduate School of Pharmaceutical Sciences, Chiba University, Chiba 260-8675, Japan

${ }^{3}$ Department of Applied Chemistry, College of Life Sciences, Ritsumeikan University, Shiga 5258577, Japan

${ }^{4}$ Research Institute for Interdisciplinary Science, Okayama University, Okayama 700-8530, Japan

*Corresponding authors. E-mail: moritat@faculty.chiba-u.jp, or ken-h@faculty.chiba-u.jp, or sumi@okayama-u.ac.jp

\section{Experimental details}

\section{Preparation of sample solutions.}

Cyclosporin A (CyA) (> 99\% purity) was purchased from LC Laboratories (Woburn, USA) and poloxamer 407 (P407, also known as Pluronic ${ }^{\circledR}$ F127 or Lutrol ${ }^{\circledR}$ F127) of pharmaceutical grade was a gift from BASF Japan Ltd. (Tokyo, Japan). The CyA as received was in amorphous state. Purified water was obtained from a Milli-Q apparatus (Millipore SAS, France). All chemicals used in this study were of reagent grade. Each amount of P407 powder (50 1000 mg) and excess 
amount of CyA powder $(10 \mathrm{mg})$ were put into the purified water of $10 \mathrm{~mL}$. Each solution was incubated for 24 hours at $25{ }^{\circ} \mathrm{C}$ using a rotating mixer. The sample solution at the P407 concentration of $0.5-10 \mathrm{wt} \%$ with or without $\mathrm{CyA}$ was obtained by filtering with a cellulose acetate membrane $0.45 \mu \mathrm{m}$ filter. The sample solutions were stable for at least one month considering repeatability and no change of DLS signals for the period, which is consistent with the present results discussed in Figure 3.

\section{SAXS measurement}

SAXS measurements were carried out using synchrotron radiation at the BL-6A station ${ }^{1}$ of the Photon Factory (PF) at the High Energy Accelerator Research Organization (KEK), Tsukuba. Point collimated X-rays were focused and monochromated using a bent mirror and a bent monochromator. The SAXS signals were acquired using a two-dimensional semiconductor-type detector, PILATUS 1M, (DECTRIS Ltd.). The distance from the sample to the detector was set at $2.5 \mathrm{~m}$ and its correct value was determined by calibration using diffraction pattern of silver behenate, AgBh. Sample holder was made of stainless steel (SUS 304). Sample length of the sample holder was set at $1.6 \mathrm{~mm}$. A pair of single crystal diamond disks (Sumitomo Electric Hardmetal Co.) with thickness $0.3 \mathrm{~mm}$ and diameter $4.0 \mathrm{~mm}$, were used for the X-ray windows. It was confirmed that damage of the sample by the incident X-rays was negligible during the exposure. After every exposure, the sample holder with single-crystal diamond windows was disassembled and carefully cleaned by polishing using cotton swab. Temperature of the sample solutions was controlled at $25^{\circ} \mathrm{C}$ by circulating water. 


\section{S(q) for neat $P 407$ micelles}

Figure S1 shows the structure factor $S(q)$ between the neat P407 micelles. Feitosa et al. have reported the $S(q)$ for 4.5 and $9.0 \mathrm{wt} \%$ of neat F127 (P407), ${ }^{2}$ the same polymeric micelle studied here. GIFT analysis and the Percus-Yevick approximation were applied to the investigation. ${ }^{2}$ Although the present evaluation method is different from the literature by Feitosa et al, the results for P407 system shown in Figure S1 are well consistent with the literature results. We referred the structure information of micelle itself reported by literature. ${ }^{2-7}$ In general, $S(q)$ is resolved by comparison between the form factor obtained by SAXS in dilute region and the total SAXS profile, as examined in our previous paper. ${ }^{8}$ However, we could not solve $S(q)$ from the procedure because SAXS of the present system changed depending on the concentration. In the present study, the $S(q)$ s were obtained on the basis of information about the referred form factor ${ }^{2-}$ 7 and interference effect using SAXS at the same concentration. The P407 micelle size in diameter was evaluated to be $19.1,18.6,18.5,18.5,18.3,18.2,18.2$, and $18.2 \mathrm{~nm}$ as a spherical micelle for $3,4,5,6,7,8,9$, and $10 \mathrm{wt} \%$ in the present measurements, respectively.

\section{Theoretical details}

\section{The MPF theory}

If precise $S(q)$ value over the full $q$-range, including the high $q$-region, is applied to inverse Fourier transform, we can directly obtain a pair correlation function $h(r)$, by which the interaction potential surface is evaluated. Namely, $h(r)$ is calculated by the inverse Fourier transform of $S(q)$ $=1+n_{0} \hat{h}(q)$, where $n_{0}$ is the number density of the particles and $\hat{h}(q)$ is the Fourier transform of $h(r)$. Unfortunately, in general, it is unable to perform the transformation using the experimental 
$S(q)$, because the $S(q)$ especially in the high $q$-region is not available as required for the transformation.

To avoid this issue, specific model-potential functions, such as the DLVO model, have typically been applied to extract $h(r)$ or the pair distribution function $g(r)=h(r)+1$ from the experimental $S(q)$. However, in complex systems, solving the interaction potential surfaces based on the model-potential method will be confronted by many unknown parameters.

In the present method, no specific model-potential functions are introduced. Instead of introduction of model-potential functions, we apply random phase approximation (RPA), as an additional closure to the Ornstein-Zernike integral equation, which explicitly consists of a basic closure relation, namely hyper-netted chain approximation or hyper-netted chain plus a bridge function $B F(r)$ as follows:

$h(r)=\exp \left[-V(r) / k_{\mathrm{B}} T+h(r)-c(r)+B F(r)\right]-1$,

where $k_{\mathrm{B}}$ is the Boltzmann constant, $T$ is the temperature, and $c(r)$ is the direct correlation function. In addition, we introduce a reference term for the hard-sphere (HS) with diameter $d_{\mathrm{HS}}$ based on the size of colloidal nanoparticle (NP), which was experimentally determined from the obtained SAXS profile. Both $V(r)$ and $c(r)$ are divided into two parts, namely the HS and the excess via $V^{\mathrm{EX}}(r)=V(r)-V^{\mathrm{HS}}(r)$ and $c^{\mathrm{EX}}(r)=c(r)-c^{\mathrm{HS}}(r)$, respectively. If we apply RPA, $c^{\mathrm{EX}}(r)=-V^{\mathrm{EX}}(r) / k_{\mathrm{B}} T$, for the additional closure equation and the HS term for the reference, we can obtain the equation (A1) as a form that does not explicitly include any model-potential functions, $V^{\mathrm{EX}}(r)$. To determine an appropriate $V(r)$ for the colloidal NPs, ${ }^{8}$ we used the following relation between $V(r)$ and $g(r)$ :

$V(r) / k_{\mathrm{B}} T=-\ln g(r)+[h(r)-c(r)+B F(r)]$. 
The validity of this equation in colloidal NP systems has been demonstrated in our previous work. ${ }^{8}$ The description of the model-potential-free scheme and computational details are provided below.

\section{Iteration procedure for the model-potential-free analysis.}

An initial guess in the iterative calculation is given by the following:

$\hat{c}(q)=\hat{c}^{H S}(q)$,

$\hat{\gamma}_{s}(q)=\hat{\gamma}_{s}^{H S}(q)=0$,

$\hat{h}_{\text {old }}(q)=\hat{h}^{H S}(q)$,

$\hat{h}^{\prime}(q)=\left\{\begin{array}{cc}\hat{h}_{\text {exp }}(q)=\left[S_{\text {exp }}(q)-1\right] / n_{0} & q \leq q_{h} \\ \hat{h}^{H S}(q) & q>q_{h}\end{array}\right.$,

where $S_{\text {exp }}(q)$ is the experimental $S(q)$, and $n_{0}$ is the number density of the colloidal particle, and $\hat{h}^{H S}(q)$ is obtained from the hard-sphere reference system with $n_{0}$. The iterative calculation is continued until the value of $S(q)=1+n_{0} \hat{h}(q)$ is equal or very close to $S_{\text {exp }}(q)$ at a small $q$.

Step $1 \hat{c}^{E X}(q)=\hat{c}(q)-\hat{c}^{H S}(q)$.

Step $2 \hat{c}(q)=\hat{h}^{\prime}(q)-\left[\hat{\gamma}_{s}(q)-\hat{c}^{E X}(q)\right]$.

Step $3 \hat{\gamma}_{s}(q)=\hat{c}(q) /\left[1-n_{0} \hat{c}(q)\right]-\hat{c}^{H S}(q)$.

Step $4 \hat{\gamma}_{s}(q)=\alpha \hat{\gamma}_{s}(q)+(1-\alpha) \hat{\gamma}_{s}^{\text {old }}(q)$,

where $\alpha$ is the dumping parameter.

Step $5 \hat{\gamma}_{s}^{\text {old }}(q)=\hat{\gamma}_{s}(q)$.

Step $6 h(r)=\left\{\begin{array}{cc}\exp \left[\gamma_{S}(r)+B F(r)\right]-1 & r>d_{H S} \\ -1 & r \leq d_{H S}\end{array}\right.$

Step 7 If the product of the difference $\Delta_{n}=S(q)-S_{\text {exp }}(q)$ for the smallest $q$ determined at $n^{\text {th }}$ iterative calculation and $\Delta_{n-1}$ determined at $(n-1)^{\text {th }}$ iterative calculation becomes negative (namely, the small angle-part of $S(q)$ becomes close to that of $S_{\text {exp }}(q)$ ), go to step 8 
(outside the loop of the iterative calculation); otherwise, we update $\hat{h}^{\prime}(q)$ using Eq. (B11) and go back to step 1 .

$$
\hat{h}^{\prime}(q)=\left\{\begin{array}{cc}
\hat{h}_{\exp }(q)=\left[S_{\text {exp }}(q)-1\right] / n_{0} & q \leq q_{h} \\
\hat{h}(q) & q>q_{h}
\end{array}\right.
$$

Step $8 S(q)$ is calculated via $S(q)=1+n_{0} \hat{h}(q)$ and $V(r)$ is calculated using

$$
V(r) / k_{B} T=-\ln g(r)+[h(r)-c(r)+B F(r)] .
$$

\section{Computational details of the MPF analysis.}

To reproduce $S_{\text {exp }}(q)$ for the polymeric micelle solutions P407 and P407 with CyA, we determined an optimal number density of the polymeric micelle $n_{0}$ by varying it as a parameter. The integral equation described above was solved using the Verlet-modified bridge function $B F(r),{ }^{9}, 10$ The maximum radial distance is $300 \mathrm{~nm}$, which is discretized by 4096 grid points. We used $0.979 \mathrm{~nm}^{-1}$ as $q_{\mathrm{h}}$ (see Eq. B11) for both the P407 and P407 with CyA. The number densities $n_{0}$ determined for the P407 and P407 with CyA are listed in Table S1.

\section{Coarse-grained modeling for polymeric micelle solution}

We modeled the polymeric micelle solutions consisted of triblock copolymer and solvent as a mixture of colloidal NP and coarse-grained polymer chain, namely, a system of the NPs immersed in a polymer solution with an implicit solvent. The coarse-grained polymer was a freely-jointed chain model consisted of twenty same segments connected with a constant length $L_{p}$, which was determined so that the radius of gyration $R_{g}=\left(N_{p} / 6\right)^{1 / 2} L_{p}$ was equal to the experimental value, $2.73 \mathrm{~nm}(=3.0 \mathrm{~nm} \times 0.91) .{ }^{4}$ The interaction potential between the segments 
of the polymer $v_{p p}(r)$ and between the colloidal NP and the segment and $v_{p c}(r)$ were given by the Lennard-Jones potential,

$v_{i j}^{L J}(r)=4 \varepsilon_{i j}\left[\left(d_{i j} / r\right)^{12}-\left(d_{i j} / r\right)^{6}\right]$

On the other hand, that between the colloidal particles $v_{c c}(r)$ was given by the Weeks-ChandlerAnderson (WCA) repulsive potential ${ }^{11}$,

$v_{i j}^{W C A}(r)=\left\{\begin{array}{cc}v_{i j}^{L J}(r)+\varepsilon_{i j} & r \leq r_{\min }=2^{1 / 6} d_{i j}, \\ 0 & r>r_{\text {min }} .\end{array}\right.$

The interaction parameters were kept fixed at the values listed in Table S2 for all the numerical calculations of the polymer concentration dependent $S(q)$. The diameter for the polymeric micelle was chosen based on the SAXS result. In the present study, we applied a density functional theory (DFT) to the polymer-colloid mixture to determine pair distribution functions between the colloidal particles $g_{c c}(r)$, between the colloidal particle and the segment of the polymer $g_{p c}(r)$, and between the segments $g_{p p}(r)$. The DFT presented here is an extension of the DFT that we had developed for a polymer chain immersed in solvents ${ }^{12}$ to finite concentration mixture of polymer chain and colloidal particle on the basis of the DFT that we had proposed for polymeric liquids $^{13}$.

We applied the DFT to the polymeric micelles at 3, 5 and $10 \mathrm{wt} \%$ polymer concentration. The number density of the colloidal NP $n_{c}^{0}$ was fixed at the polymeric micelle value determined by the MPF analysis. Using the value of $n_{c}^{0}$ and the aggregation number of polymer chain contained in a polymer micelle 72,5 we estimated the number density of polymer chain $n_{p}^{0}$ remaining in the solution according to the conservation of total number of polymer chain in the system (Table S3). The details of the DFT and its computation are provided in the next section. 


\section{Supporting Information Tables}

Table S1. The number density of polymeric micelles $n_{c}^{0}$ determined by the MPF calculation for neat P407 micelles and CyA-entrapped $\mathrm{P} 407$ micelles at $25^{\circ} \mathrm{C}$.

\begin{tabular}{|c|c|c|}
\hline P407 concentration (wt\%) & P407 $\left(\mathrm{nm}^{-3}\right)$ & P407+ CyA $\left(\mathrm{nm}^{-3}\right)$ \\
\hline 3 & $7.45 \times 10^{-6}$ & $9.65 \times 10^{-6}$ \\
\hline 4 & $1.58 \times 10^{-5}$ & $1.93 \times 10^{-5}$ \\
\hline 5 & $2.45 \times 10^{-5}$ & $2.70 \times 10^{-5}$ \\
\hline 6 & $3.55 \times 10^{-5}$ & $3.50 \times 10^{-5}$ \\
\hline 7 & $4.07 \times 10^{-5}$ & $4.17 \times 10^{-5}$ \\
\hline 8 & $5.08 \times 10^{-5}$ & $4.90 \times 10^{-5}$ \\
\hline 9 & $5.90 \times 10^{-5}$ & $6.20 \times 10^{-5}$ \\
\hline 10 & $6.32 \times 10^{-5}$ & $6.80 \times 10^{-5}$ \\
\hline
\end{tabular}

$n_{c}^{0}$ determined by Feitosa et al. for $9 \mathrm{wt} \% \mathrm{~F} 127$ without and with loading $\mathrm{HePC}$ at $37^{\circ} \mathrm{C}$ was evaluated to be $5.21 \times 10^{-5}$ and $5.73 \times 10^{-5} \mathrm{~nm}^{-3}$, respectively. ${ }^{2}$

Table S2. The potential parameters for the mixture of colloidal NP

\begin{tabular}{|l|l|l|l|}
\hline & \multicolumn{1}{|c|}{$v_{c c}(r)$} & \multicolumn{1}{c|}{$v_{p c}(r)$} & \multicolumn{1}{c|}{$v_{p p}(r)$} \\
\hline$\varepsilon_{i j}(\mathrm{kcal} / \mathrm{mol})$ & 0.4286 & 0.0320 & 0.0700 \\
\hline$d_{i j}(\mathrm{~nm})$ & 18.0 & 9.96 & 1.92 \\
\hline
\end{tabular}

Table S3. The number density of the colloidal NP $n_{c}^{0}$ and that of the polymer chain remaining in the solution $n_{p}^{0}$.

\begin{tabular}{|l|l|l|l|}
\hline & $3 \mathrm{wt} \%$ & $5 \mathrm{wt} \%$ & $10 \mathrm{wt} \%$ \\
\hline$n_{c}^{0}\left(\mathrm{~nm}^{-3}\right)^{\mathrm{a}} \%$ & $7.45 \times 10^{-6}$ & $2.45 \times 10^{-5}$ & $6.32 \times 10^{-5}$ \\
\hline$n_{p}^{0}\left(\mathrm{~nm}^{-3}\right)^{\mathrm{b})}$ & $9.09 \times 10^{-4}$ & $6.45 \times 10^{-4}$ & $2.67 \times 10^{-4}$ \\
\hline
\end{tabular}

a) The values were determined by the MPF analysis (Table S1). ${ }^{\text {b) }}$ We assumed 72 as the aggregation number of polymer chain contained in a polymer micelle. ${ }^{5}$ 


\section{Supporting Information Figures}

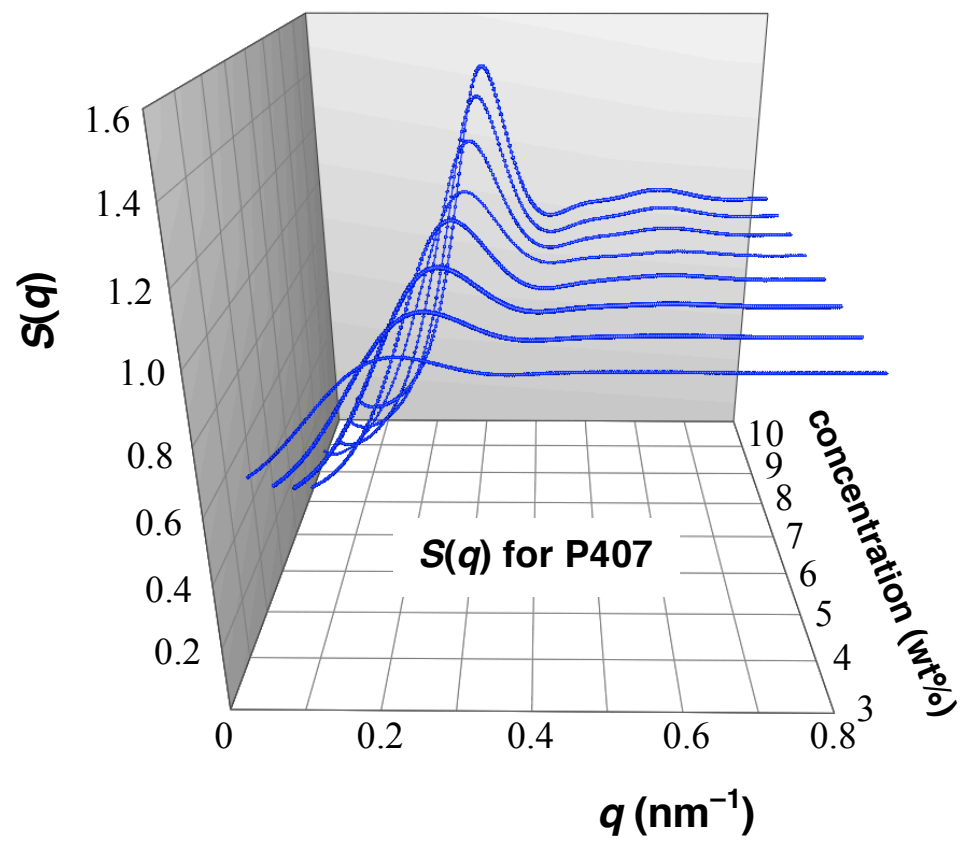

Figure S1. Concentration dependence of the evaluated $S(q)$ between the neat P407 micelles in $\mathrm{H}_{2} \mathrm{O}$ at $25{ }^{\circ} \mathrm{C}$. 

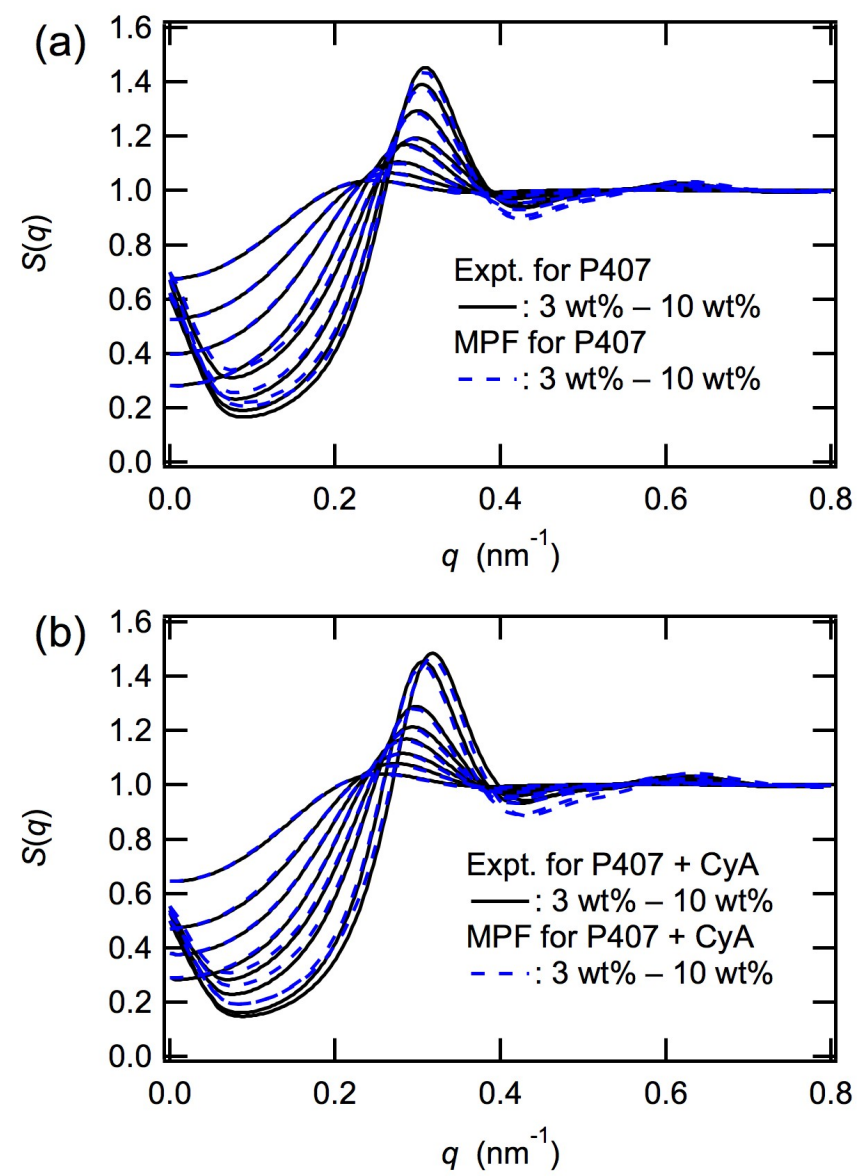

Figure S2. Comparison between the experimental $S(q)$ and the $S(q)$ obtained from the MPF calculation at 3, 4, 5, 6, 7, 8, 9, and $10 \mathrm{wt} \%$. (a) P407 micelles. (b) P407 micelles with CyA. 


\section{Intensity}

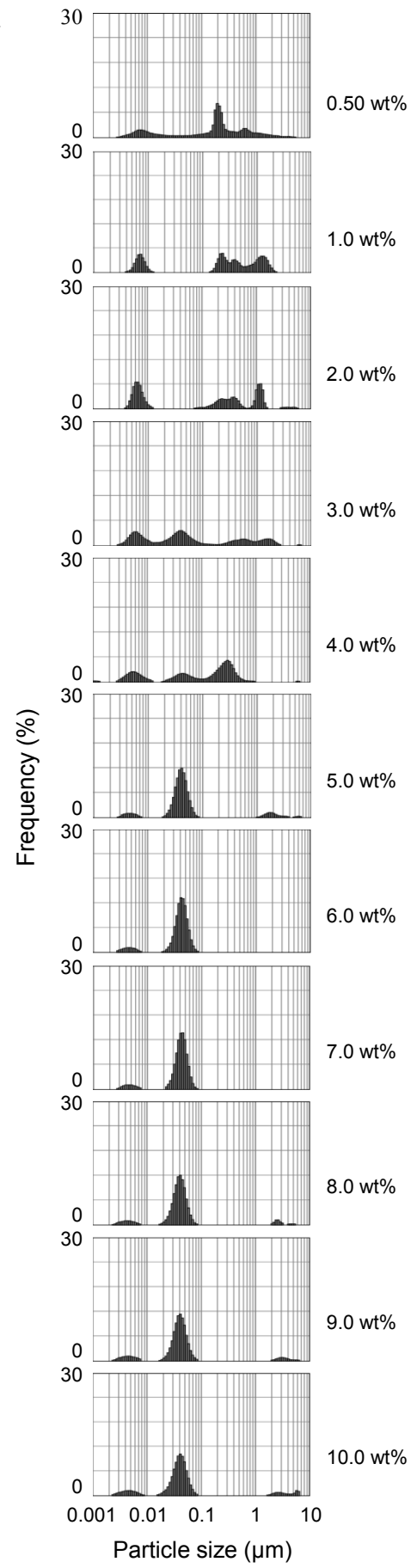

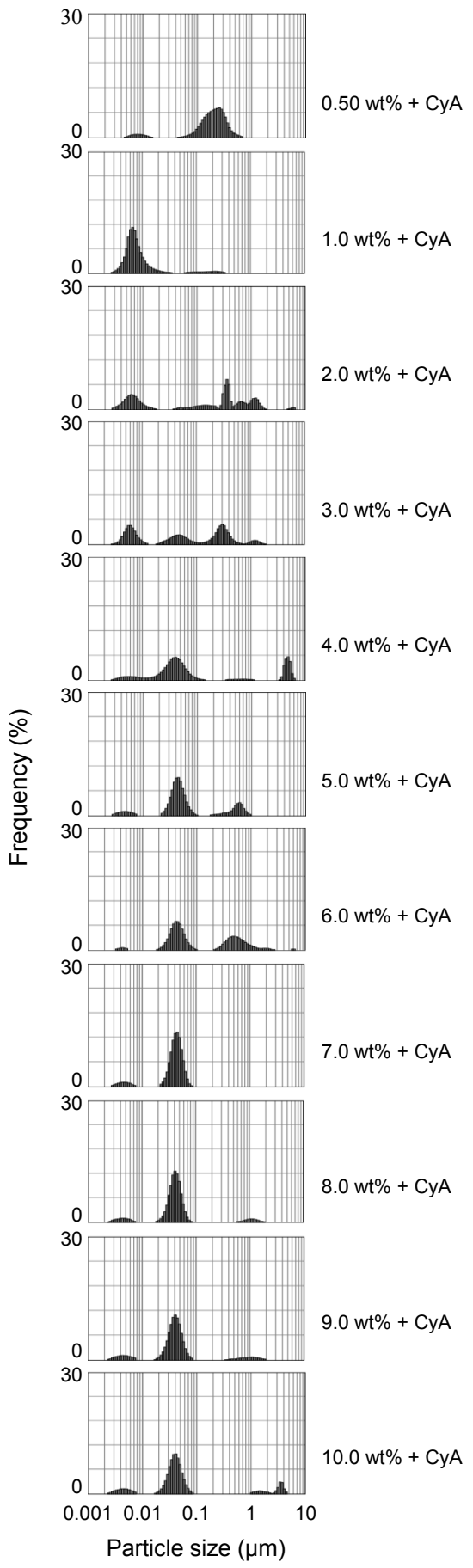

Figure S3. Intensity-weighted frequency obtained by the DLS analysis without filtering treatment immediately before the DLS measurement. 

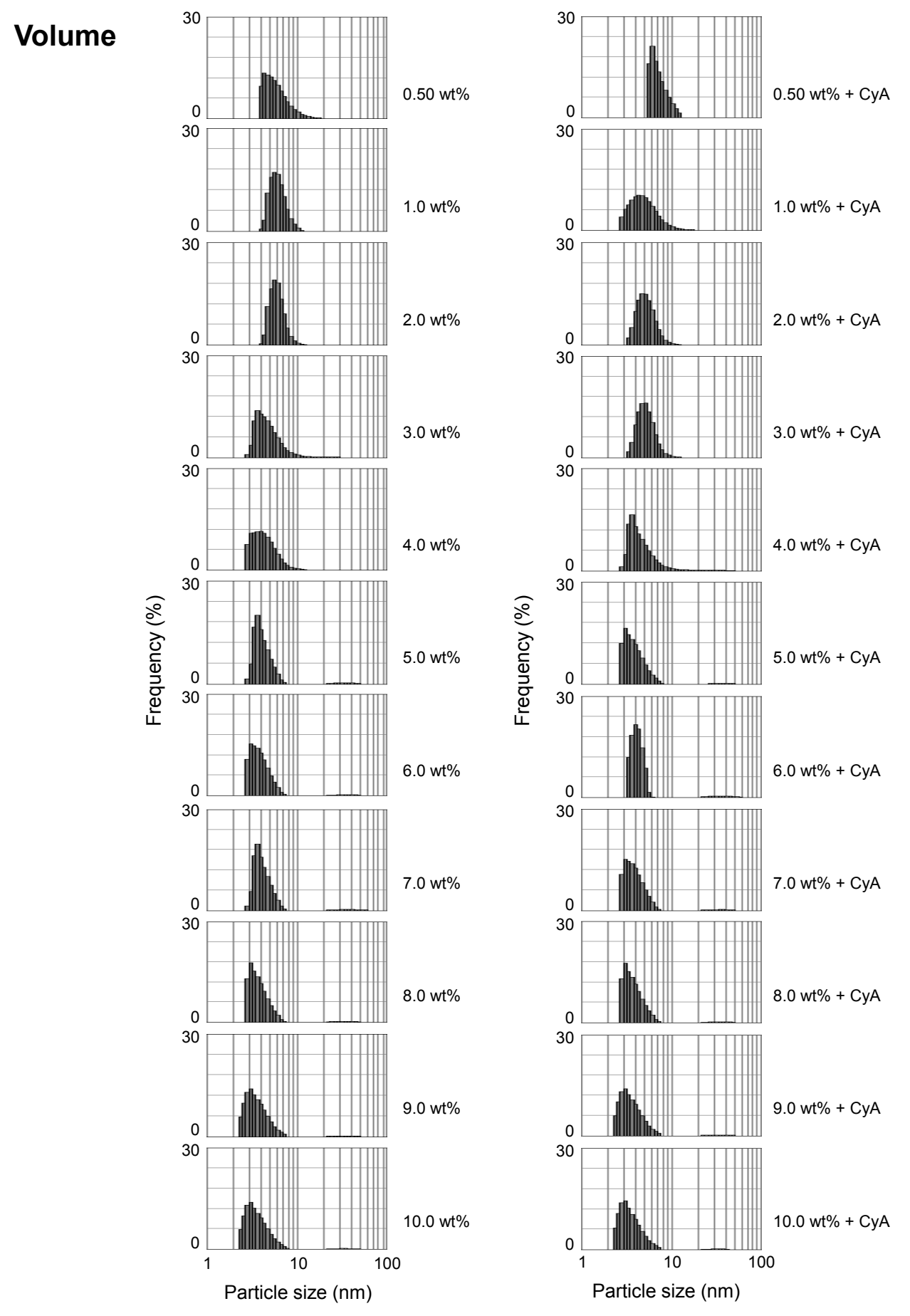

Figure S4. Volume-weighted frequency obtained by the DLS analysis without filtering treatment immediately before the DLS measurement. 

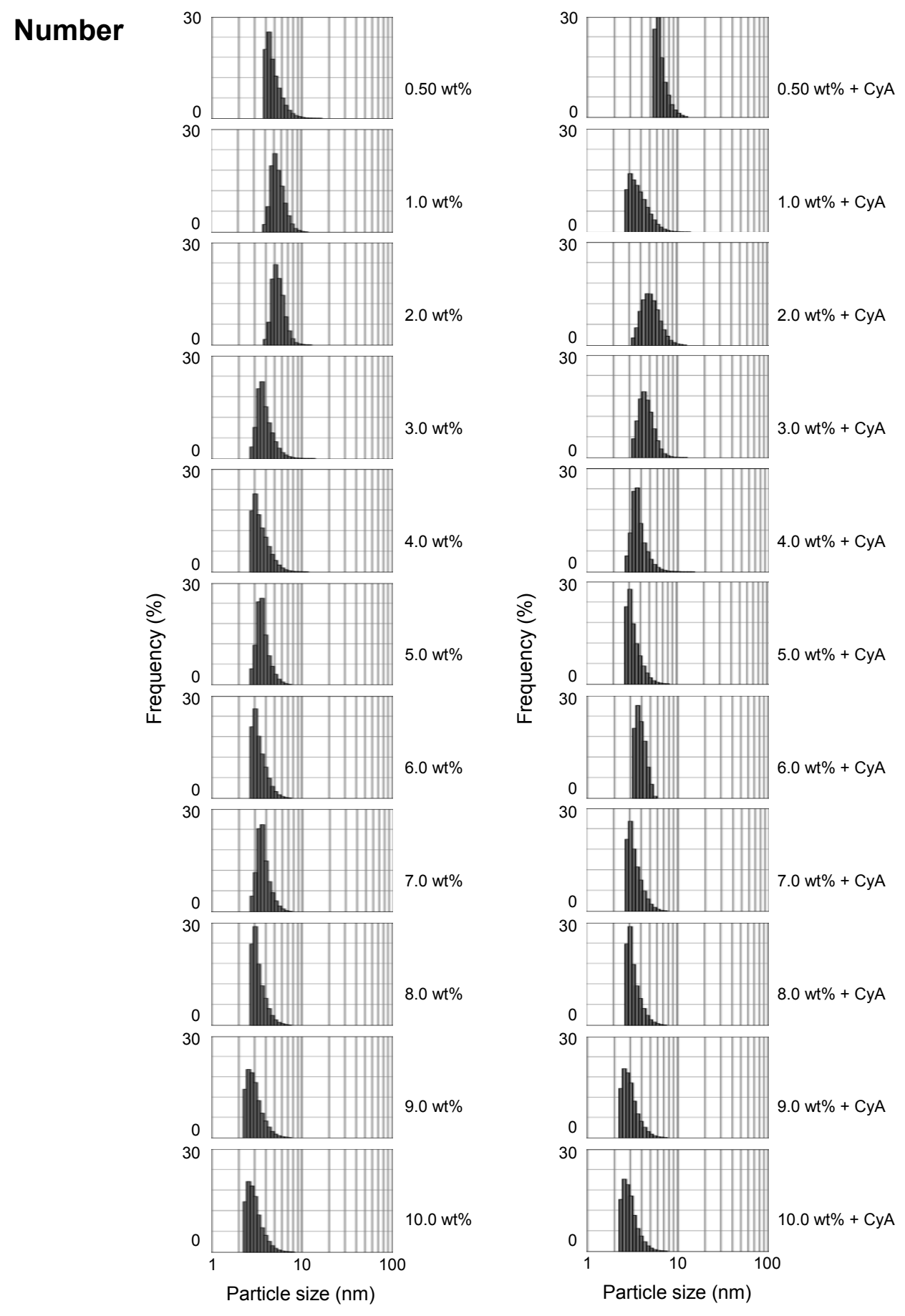

Figure S5. Number-weighted frequency obtained by the DLS analysis without filtering treatment immediately before the DLS measurement. 


\section{Intensity}

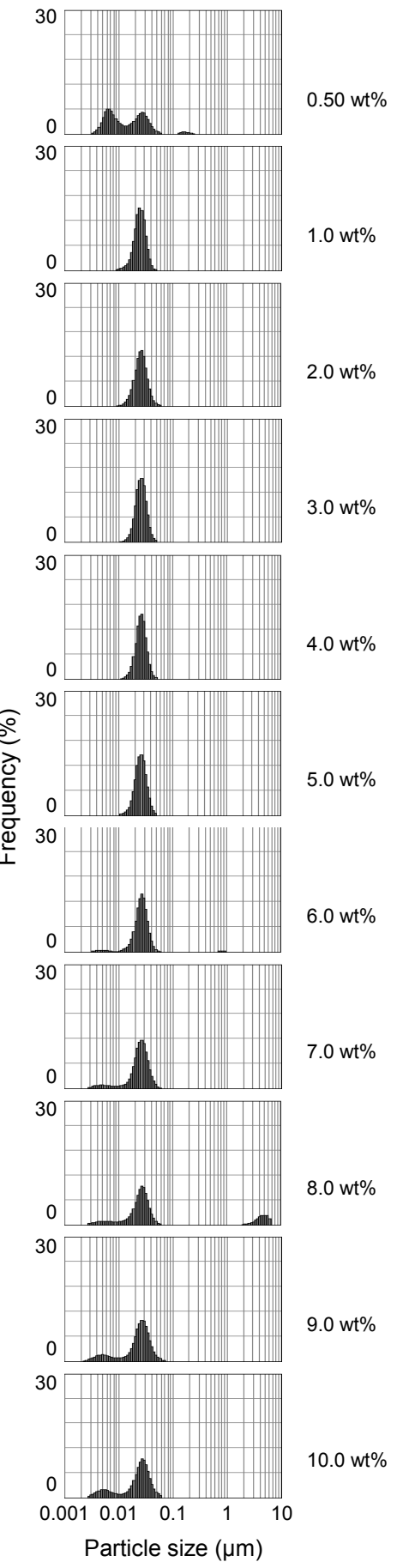

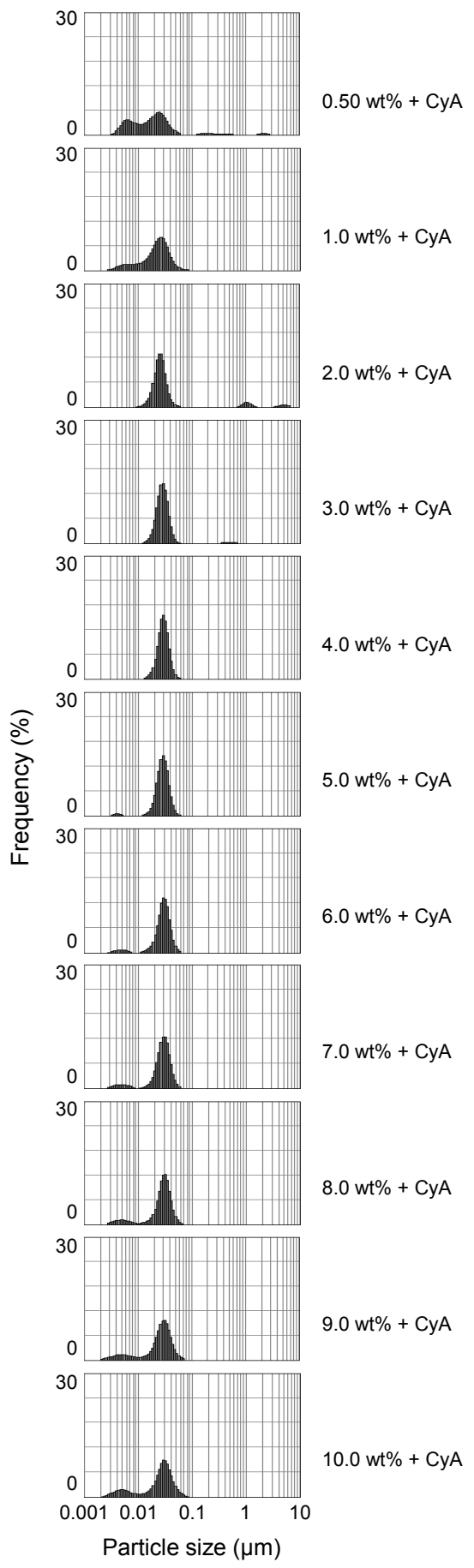

Figure S6. Intensity-weighted frequency obtained by the DLS analysis with filtering treatment immediately before the DLS measurement. 
Volume

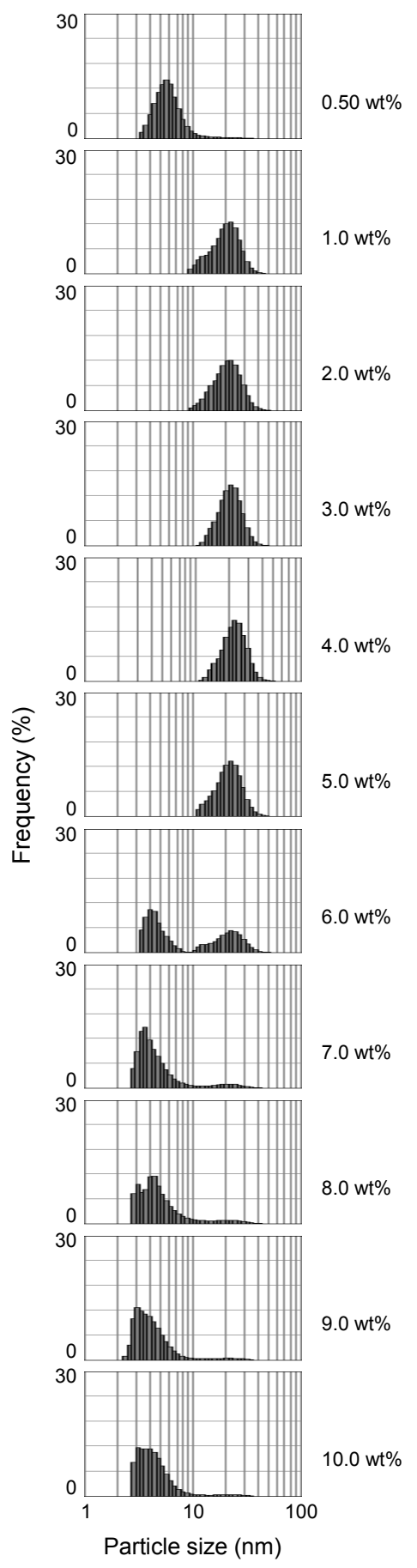

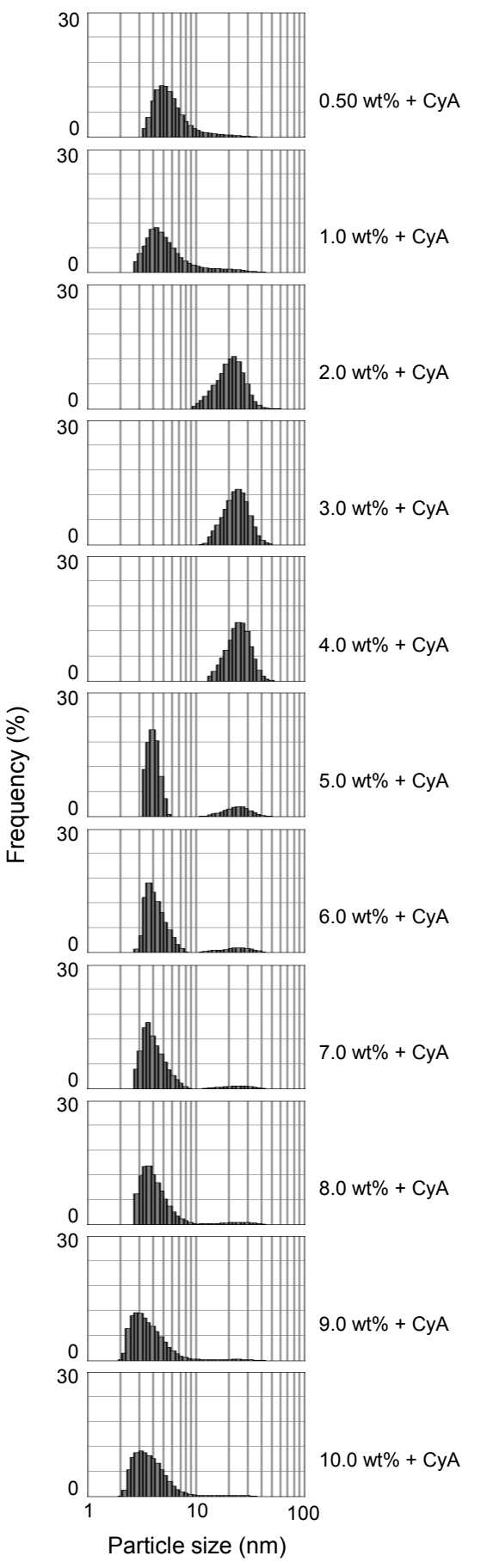

Figure S7. Volume-weighted frequency obtained by the DLS analysis with filtering treatment immediately before the DLS measurement. Frequency for micelle component became higher at 2.0, 3.0, and $4.0 \mathrm{wt} \%$ due to the complex deconvolution analysis and the micelle structure inhomogeneity. 

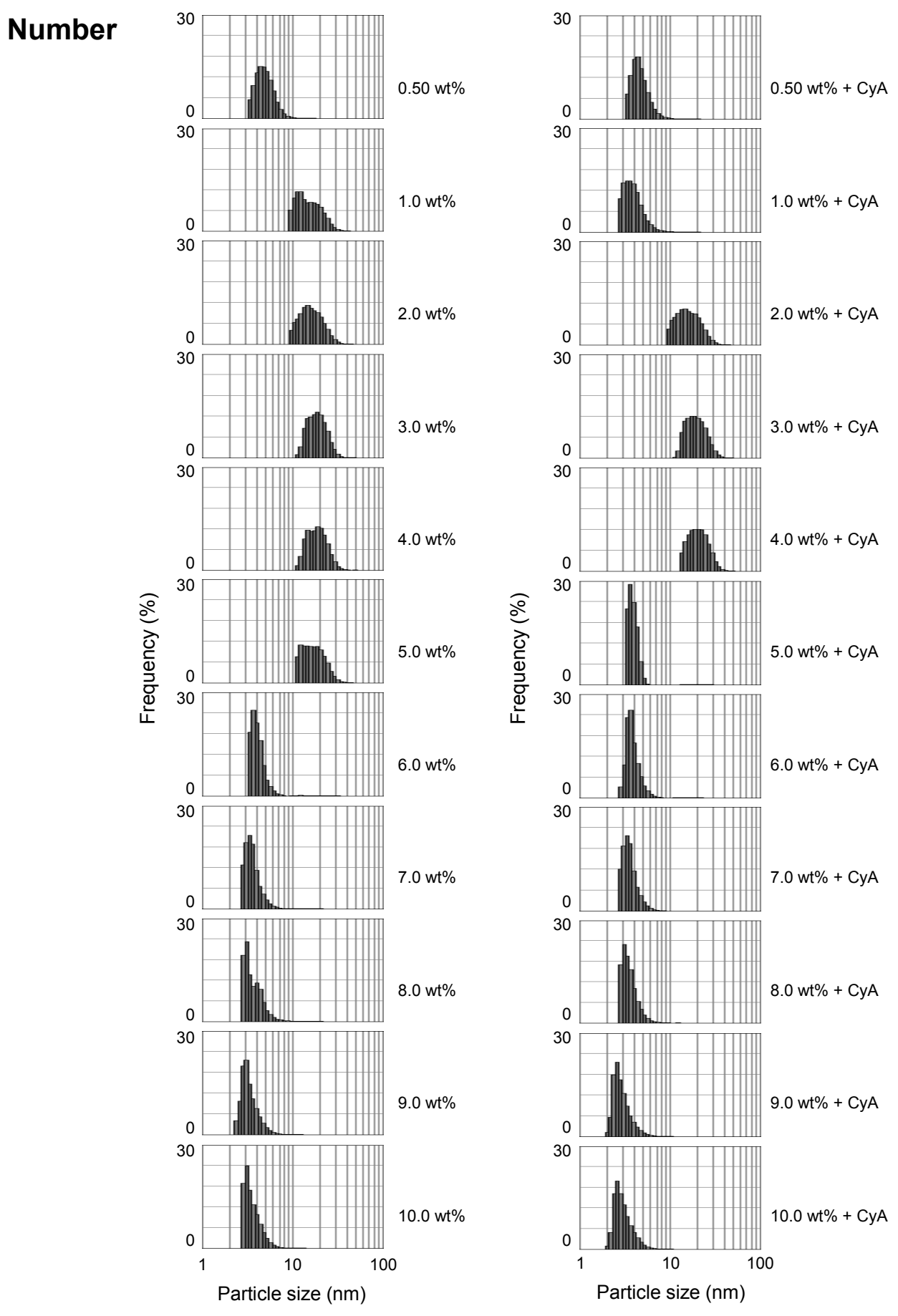

Figure S8. Number-weighted frequency obtained by the DLS analysis with filtering treatment immediately before the DLS measurement. Frequency for micelle component became higher at 2.0, 3.0, and $4.0 \mathrm{wt} \%$ due to the complex deconvolution analysis and the micelle structure inhomogeneity. 
(a)

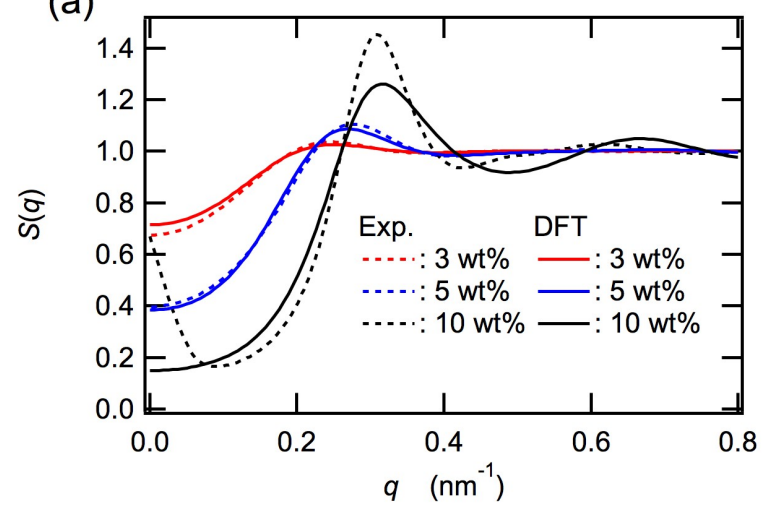

(b)
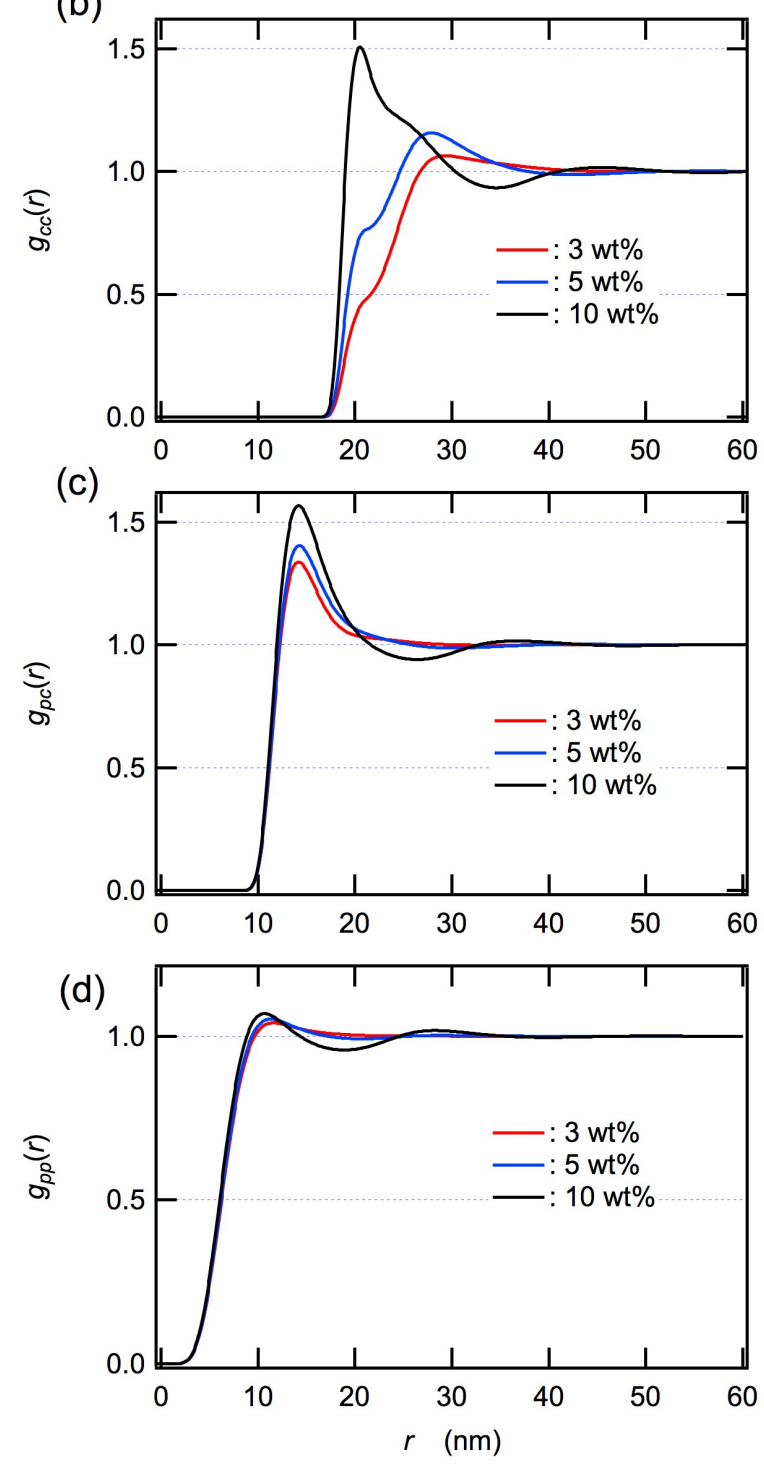

Figure S9. (a) Comparison of the structure factors $S(q)$ obtained from the DFT with the experimental $S(q)$. (b) The pair distribution functions between colloidal particles $g_{c c}(r)$. (c) The pair distribution functions between polymer chain and colloidal particle $g_{p c}(r)$. (d) The pair distribution functions between polymer chains $g_{p p}(r)$. The definition of these pair distribution functions is provided in the Supporting Information Appendix. As shown in Figure S9(a), $S(q) \mathrm{s}$ obtained from the DFT at 3 and $5 \mathrm{wt} \%$ are quantitatively consistent with the experimental $S(q) \mathrm{s}$. As shown in Figure S9(c), the colloidal NPs weakly adsorb the polymer chains. Therefore, the repulsive force between the colloidal particles is interpreted by effects of the polymer chains. The first peak in $S(q)$ as well as $S(q=0)$ underestimated by the DFT at $10 \mathrm{wt} \%$ as shown in Figure S9(a) are attributed to lack of the long-range attractive interaction in $V(r)$. 


\section{Supporting Information Appendix}

\section{Density functional theory for mixtures of colloid and polymer chain}

By using the density functional theory (DFT) for polymer liquid/solution, which we had presented $^{12,13}$, we developed an integral equation theory for a mixture of colloidal NP and polymer chain to investigate the structure of polymeric micelle solution consisted of triblock copolymer and solvent. In our approach, we assumed that the polymeric micelle is modeled as the colloidal NP and triblock copolymer remained in the solvent is modeled as a freely-joined chain consisted of 20 same coarse-grained segments. Here, we avoid to show the details of the derivation on integral equations based on the DFT, while we provide the final expression of the integral equations which is useful for implementation to calculate pair correlation functions. We introduced the following correlation functions:

$\left(n_{c}^{0}\right)^{2}\left[h_{c c}\left(\left|\mathbf{R}-\mathbf{R}^{\prime}\right|\right)+1\right]=\left\langle\sum_{k \neq l}^{N_{c}} \delta\left(\left|\mathbf{R}-\mathbf{R}_{k}\right|\right) \delta\left(\left|\mathbf{R}^{\prime}-\mathbf{R}_{l}\right|\right)\right\rangle$

$n_{p}^{0} n_{c}^{0}\left[h_{p c}\left(\left|\mathbf{r}-\mathbf{R}^{\prime}\right|\right)+1\right]=\left(\frac{1}{P}\right) \sum_{a=1}^{P}\left\langle\sum_{k=1}^{N_{p}} \sum_{l=1}^{N_{c}} \delta\left(\left|\mathbf{r}-\mathbf{r}_{\boldsymbol{k}}^{a}\right|\right) \delta\left(\left|\mathbf{R}^{\prime}-\mathbf{R}_{l}\right|\right)\right\rangle$

$\left(n_{p}^{0}\right)^{2}\left[h_{p p}\left(\left|\mathbf{r}-\mathbf{r}^{\prime}\right|\right)+1\right]=\left(\frac{1}{P}\right)^{2} \sum_{a=1}^{P} \sum_{b=1}^{P}\left\langle\sum_{k=1}^{N_{p}} \sum_{l \neq k}^{N_{p}} \delta\left(\left|\mathbf{r}-\mathbf{r}_{\boldsymbol{k}}^{\boldsymbol{a}}\right|\right) \delta\left(\left|\mathbf{r}-\mathbf{r}_{l}^{\boldsymbol{b}}\right|\right)\right\rangle$,

$n_{p}^{0} \sigma_{p p}\left(\left|\mathbf{r}-\mathbf{r}^{\prime}\right|\right)=\left(\frac{1}{P}\right)^{2} \sum_{a=1}^{P} \sum_{b=1}^{P}\left\langle\sum_{k=1}^{N_{p}} \delta\left(\left|\mathbf{r}-\mathbf{r}_{\boldsymbol{k}}^{\boldsymbol{a}}\right|\right) \delta\left(\left|\mathbf{r}-\mathbf{r}_{\boldsymbol{k}}^{\boldsymbol{b}}\right|\right)\right\rangle$,

where $N_{c}, N_{p}$, and $P$ are the number of colloidal particles, polymer chains, and segments on the polymer chain; $n_{c}^{0}$ and $n_{p}^{0}$ are the number density of colloidal particle and polymer chain, respectively. $h_{c c}(r), h_{p c}(r)$, and $h_{p p}(r)$ are the pair correlation function between colloidal particles, between segment of polymer chain and colloidal particle, and between polymer segments, respectively. $\sigma_{p p}(r)$ is the intramolecular correlation function between segments of a polymer chain. For instance, the radial distance $r$ in $\sigma_{p p}(r)$ indicates the intramolecular distance 
between segments of a polymer chain, and the radial distance $r$ in $h_{p p}(r)$ indicates the intermolecular distance between segments of different polymer chains. \langle\rangle is the ensemble average provided by the ground canonical partition function for the mixture of colloid and polymer chain without external field. These pair correlation functions were calculated from the following integral equations:

$$
\begin{aligned}
& g_{c c}\left(\left|\mathbf{R}-\mathbf{R}^{\prime}\right|\right) \equiv h_{c c}\left(\left|\mathbf{R}-\mathbf{R}^{\prime}\right|\right)+1=\exp \left(-\beta v_{c c}^{e f f}\left(\left|\mathbf{R}-\mathbf{R}^{\prime}\right|\right)\right) \\
& g_{p c}\left(\left|\mathbf{r}-\mathbf{R}^{\prime}\right|\right) \equiv h_{p c}\left(\left|\mathbf{r}-\mathbf{R}^{\prime}\right|\right)+1 \\
& =\left[\left(\frac{1}{P}\right) \sum_{d=1}^{P} \int\left\{\prod_{a=1}^{P} d \mathbf{r}_{1}^{a}\right\} \delta\left(\left|\mathbf{r}-\mathbf{r}_{\mathbf{1}}^{\boldsymbol{d}}\right|\right)\left\{\prod_{b=1}^{P-1} s\left(\left|\mathbf{r}_{\mathbf{1}}^{\boldsymbol{b}}-\mathbf{r}_{\mathbf{1}}^{\boldsymbol{b}+\mathbf{1}}\right|\right)\right\}\left\{\prod_{b=1}^{P} \exp \left(-\beta v_{p c}^{e f f}\left(\left|\mathbf{r}_{\mathbf{1}}^{\boldsymbol{b}}-\mathbf{R}^{\prime}\right|\right)\right)\right\}\right] \\
& \quad \times\left[\left(\frac{1}{P}\right) \sum_{d=1}^{P} \int\left\{\prod_{a=1}^{P} d \mathbf{r}_{1}^{a}\right\} \delta\left(\left|\mathbf{r}-\mathbf{r}_{\mathbf{1}}^{\boldsymbol{d}}\right|\right)\left\{\prod_{b=1}^{P-1} s\left(\left|\mathbf{r}_{\mathbf{1}}^{\boldsymbol{b}}-\mathbf{r}_{\mathbf{1}}^{\boldsymbol{b}+\mathbf{1}}\right|\right)\right\}\right]^{-1} \\
& g_{p p}\left(\left|\mathbf{r}-\mathbf{r}^{\prime}\right|\right) \equiv h_{p p}\left(\left|\mathbf{r}-\mathbf{r}^{\prime}\right|\right)+1 \\
& \quad=\left[\left(\frac{1}{P}\right) \sum_{d=1}^{P} \int\left\{\prod_{a=1}^{P} d \mathbf{r}_{1}^{a}\right\} \delta\left(\left|\mathbf{r}^{\prime}-\mathbf{r}_{\mathbf{1}}^{\boldsymbol{d}}\right|\right)\left\{\prod_{b=1}^{P-1} s\left(\left|\mathbf{r}_{\mathbf{1}}^{\boldsymbol{b}}-\mathbf{r}_{\mathbf{1}}^{\boldsymbol{b}+\mathbf{1}}\right|\right)\right\}\left\{\prod_{b=1}^{P} \theta_{p}\left(\left|\mathbf{r}-\mathbf{r}_{\mathbf{1}}^{\boldsymbol{b}}\right|\right)\right\}\right] \\
& \times\left[\left(\frac{1}{P}\right) \sum_{d=1}^{P} \int\left\{\prod_{a=1}^{P} d \mathbf{r}_{1}^{a}\right\} \delta\left(\left|\mathbf{r}-\mathbf{r}_{\mathbf{1}}^{\boldsymbol{d}}\right|\right)\left\{\prod_{b=1}^{P-1} s\left(\left|\mathbf{r}_{\mathbf{1}}^{\boldsymbol{b}}-\mathbf{r}_{\mathbf{1}}^{\boldsymbol{b + 1}}\right|\right)\right\}\right]^{-1}
\end{aligned}
$$

where

$\prod_{b=1}^{P} \theta_{p}\left(\left|\mathbf{r}-\mathbf{r}_{1}^{b}\right|\right) \equiv \prod_{b=1}^{P}\left[\left(\frac{1}{P}\right) \sum_{c=1}^{P} \int\left\{\prod_{a=1}^{P} d \mathbf{r}_{2}^{a}\right\} \delta\left(\left|\mathbf{r}-\mathbf{r}_{2}^{c}\right|\right)\left\{\prod_{b=1}^{P-1} s\left(\mid \mathbf{r}_{2}^{b}-\right.\right.\right.$

$\left.\left.\left.\mathbf{r}_{2}^{b+1} \mid\right)\right\}\left\{\prod_{a=1}^{P-1} \exp \left(-\beta v_{p p}^{e f f}\left(\left|\mathbf{r}_{2}^{a}-\mathbf{r}_{1}^{b}\right|\right)\right)\right\}\right]$,

$v_{\alpha \gamma}^{e f f}\left(\left|\mathbf{r}-\mathbf{r}^{\prime}\right|\right) \equiv v_{\alpha \gamma}\left(\left|\mathbf{r}-\mathbf{r}^{\prime}\right|\right)-\frac{1}{\beta} \Gamma_{\alpha \gamma}\left(\left|\mathbf{r}-\mathbf{r}^{\prime}\right|\right)$,

$\Gamma_{\alpha \gamma}\left(\left|\mathbf{r}-\mathbf{r}^{\prime}\right|\right) \equiv \sum_{I}^{c, p} \sum_{J}^{c, p} \int d \mathbf{r}_{1} d \mathbf{r}_{2} C_{\alpha I}\left(\left|\mathbf{r}-\mathbf{r}_{1}\right|\right) n_{I}^{0} h_{I J}\left(\left|\mathbf{r}_{1}-\mathbf{r}_{2}\right|\right) \sigma_{J \gamma}^{-1}\left(\left|\mathbf{r}_{2}-\mathbf{r}^{\prime}\right|\right) P_{\gamma}^{-1}$

where $\alpha$ and $\gamma$ are the component polymer $(p)$ or colloid $(c)$. In these equations, $\beta=1 / k_{B} T$ with the Boltzmann constant $k_{B} ; s\left(\left|\mathbf{r}_{\mathbf{1}}^{\boldsymbol{b}}-\mathbf{r}_{\mathbf{1}}^{\boldsymbol{b}+\mathbf{1}}\right|\right)$ is the bonding function between segments of the polymer chain with the bond length $L_{p}$ and is provided by $\delta\left(\left|\mathbf{r}_{\mathbf{1}}^{\mathbf{b}}-\mathbf{r}_{\mathbf{1}}^{\boldsymbol{b}+\mathbf{1}}\right|-L_{p}\right) / 4 \pi L_{p}^{2}$ with the 
Dirac delta function $\delta(r)$. The volume integrals with respect to the position of polymer segment appearing in Eqs. (S6)-(S8) are convolution integrals, thus we can efficiently perform these integrals using the first Fourier transformation (FFT) algorithm. ${ }^{12,13}$ In Eq. (S10), $P_{\gamma}$ is 1 for the colloid and $P$ for the polymer. $C_{\alpha \gamma}(r)$ in Eq. (S10) is the direct correlation function defined by the following generalized Ornstein-Zernike (OZ) equation:

$\mathbf{h}(k)=\boldsymbol{\sigma}(k) \mathrm{PC}(k) \mathbf{n}^{0} \boldsymbol{\sigma}(k) \mathbf{P n}^{0^{-1}}+\boldsymbol{\sigma}(k) \mathbf{P C}(k) \mathbf{n}^{0} \mathbf{h}(k)$,

where the bold forms $\mathbf{h}(k), \mathbf{C}(k)$, and $\boldsymbol{\sigma}(k)$ are the $2 \times 2$ matrixes for the Fourier transform of $h_{\alpha \gamma}(r), C_{\alpha \gamma}(r)$, and $\sigma_{\alpha \gamma}(r)$, respectively. $\sigma_{\alpha \gamma}(r)$ is equal to zero for $\alpha \neq \gamma$ and $\sigma_{c c}(r)=\delta(r)$ for the colloid; for the polymer chain, we employed of a freely-jointed chain model as $\sigma_{p p}(k)$ which is calculated using $\left[1-s(k)^{2}-2 P^{-1} s(k)\left(1-s(k)^{P}\right)\right] /(1-s(k))^{2}$ with the Fourier transform of the bonding function $s(r)^{14}$. By using Eq. (S11), we obtain the numerically tractable simple equation for (S10) as:

$\boldsymbol{\Gamma}(k)=\mathbf{P}^{-1} \boldsymbol{\sigma}^{-1}(k) \mathbf{h}(k) \boldsymbol{\sigma}^{-1}(k) \mathbf{P}^{-1}-\mathbf{C}(k)$,

which seems to be a generalization of hypernetted chain approximation for the mixture of colloid and polymer chain. All the integral equations provided by Eqs (S5)-(S12) were solved using 8192 grid points for $500 \mathrm{~nm}$ radial distance in the self-consistent manner. 


\section{References}

[1] Takagi, H.; Igarashi, N.; Mori, T.; Saijo, S.; Ohta, H.; Nagatani, Y.; Kosuge, T.; Shimizu,

N. Upgrade of Small Angle X-ray Scattering Beamline BL-6A at the Photon Factory. AIP Conf. Proc. 2016, 1741, 030018.

[2] Feitosa, V. A.; Almeida, V. C.; Malheiros, B.; Castro, R. D.; Barbosa, L. R. S.; Cerize, N. N. P.; Rangel-Yagui, C. O. Polymeric Micelles of Pluronic F127 Reduce Hemolytic Potential of Amphiphilic Drugs. Colloids Surf. B: Biointerfaces 2019, 180, 177.

[3] Valero, M.; Castiglione, F.; Mele, A.; da Silva, M. A.; Grillo, I.; González-Gaitano, G.; Dreiss, C. A. Competitive and Synergistic Interactions between Polymer Micelles, Drugs, and Cyclodextrins: The Importance of Drug Solubilization Locus. Langmuir 2016, 32, 13174.

[4] Bogomolova, A.; Hruby, M.; Panek, J.; Rabyk, M.; Turner, S.; Bals, S.; Steinhart, M.; Zhigunov, A.; Sedlacek, O.; Stepanek, P.; Filippov, S. K. Small-Angle X-ray Scattering and Light Scattering Study of Hybrid Nanoparticles Composed of Thermoresponsive Triblock Copolymer F127 and Thermoresponsive Statistical Polyoxazolines with Hydrophobic Moieties. J. Appl. Cryst. 2013, 46, 1690.

[5] Desai, P. R.; Jain, N. J.; Sharma, R. K.; Bahadur, P. Effect of Additives on the Micellization of PEO/PPO/PEO Block Copolymer F127 in Aqueous Solution. Colloids Surf. A: Physicochem. Eng. Aspects 2001, 178, 57.

[6] Basak, R.; Bandyopadhyay, R. Encapsulation of Hydrophobic Drugs in Pluronic F127 Micelles: Effects of Drug Hydrophobicity, Solution Temperature, and pH. Langmuir 2013, 29, 4350.

[7] Hamley, I. W.; Castelletto, V. Small-Angle Scattering of Block Copolymers: in the Melt, Solution and Crystal State. Prog. Polym. Sci. 2004, 29, 909. 
[8] Morita, T.; Uehara, N.; Kuwahata, K.; Imamura, H.; Shimada, T.; Ookubo, K.; Fujita, M.; Sumi, T. Interaction Potential between Biological Sensing Nanoparticles Determined by Combining Small-Angle X-ray Scattering and Model-Potential-Free Liquid Theory. J. Phys. Chem. C. 2016, 120, 25564.

[9] Verlet, L. Integral Equations for Classical Fluids I. The Hard Sphere Case. Mol. Phys. 1980, $41,183$.

[10] Choudhury, N.; Ghosh, S. K. Integral Equation Theory of Lennard-Jones Fluids: A Modified Verlet Bridge Function Approach. J. Chem. Phys. 2002, 116, 8517.

[11] Weeks, J. D.; Chandler, D.; Andersen, H. C. Role of Repulsive Forces in Determining the Equilibrium Structure of Simple Liquids. J. Chem. Phys. 1971, 54, 5237.

[12] Sumi, T.; Sekino, H. A Cooperative Phenomenon between Polymer Chain and Supercritical Solvent: Remarkable Expansions of Solvophobic and Solvophilic Polymers. J. Chem. Phys. 2005, 122, 194910.

[13] Sumi, T.; Hirata, F. A Density-Functional Theory for Polymer Liquids Based on the Interaction Site Model. J. Chem. Phys. 2003, 118, 2431.

[14] Schweizer, K. S.; Curro, J. G. Integral Equation Theory of Polymer Melts: Intramolecular Structure, Local Order, and the Correlation Hole. Macromolecules 1988, 21, 3070. 